\title{
Prognostic value of serum neurofilaments in patients with clinically isolated syndromes
}

Gloria Dalla Costa, MD, * Vittorio Martinelli, MD,* Francesca Sangalli, MD, Lucia Moiola, MD,

Bruno Colombo, MD, Marta Radaelli, MD, Letizia Leocani, MD, Roberto Furlan, MD, and Giancarlo Comi, MD

Neurology ${ }^{\circledR}$ 2019;92:e733-e741. doi:10.1212/WNL.0000000000006902

\section{Abstract}

\section{Objective}

To assess the prognostic role of serum neurofilament light chains (NfL) for clinically defined multiple sclerosis (CDMS) and McDonald 2017 multiple sclerosis (MS) in patients with clinically isolated syndromes (CIS).

\section{Methods}

We retrospectively analyzed data of patients admitted to our neurologic department between 2000 and 2015 for a first demyelinating event. We evaluated baseline serum NfL in addition to CSF, MRI, and clinical data.

\section{Results}

Among 222 patients who were enrolled (mean follow-up 100.6 months), 45 patients (20\%) developed CDMS and 141 patients (63.5\%) developed 2017 MS at 2 years. Serum NfL (median 22.0, interquartile range $11.6-40.4 \mathrm{pg} / \mathrm{mL}$ ) was noticeably increased in patients with a recent relapse, with a high number of $\mathrm{T} 2$ and gadolinium-enhancing lesions at baseline MRI. Serum NfL was prognostic for both CDMS and McDonald 2017 MS, with a threefold and a twofold respective reduction in CDMS and 2017 MS risk in those patients with low and extremely low levels of NfL. The results remained unchanged subsequent to adjustment for such established MS prognostic factors as oligoclonal bands, Gd-enhancing lesions, and a high T2 lesion load at baseline MRI. NfL was associated with disability at baseline but not at follow-up.

\section{Conclusions}

Serum NfL have a prognostic value for CIS patient conversion to MS. NfL might play a twin role as biomarker in MS as peak level measurements can act as a quantitative marker of serious inflammatory activity, while steady-state levels can be a reflection of neurodegenerative and chronic inflammatory processes.

\section{Correspondence}

Prof. Comi

comi.giancarlo@hsr.it

\section{RELATED ARTICLE}

\section{Editorial}

Serum neurofilament light and prediction of multiple sclerosis in clinically isolated syndrome

Page 313

\footnotetext{
*These authors contributed equally to this work.

From the Department of Neurology (G.D.C., V.M., F.S., L.M., B.C., M.R., G.C.), Experimental Neurophysiology Research Unit (L.L.), and Neuroimmunology Research Unit (R.F.), San Raffaele Hospital, Milan, Italy.

Go to Neurology.org/N for full disclosures. Funding information and disclosures deemed relevant by the authors, if any, are provided at the end of the article. The Article Processing Charge was funded by a project granted by the Italian Ministry of Health RF-2011-02349698. 


\section{Glossary}

c-index = concordance index CDMS = clinically definite multiple sclerosis; $\mathbf{C I}=$ confidence interval; $\mathbf{C I S}=$ clinically isolated syndromes; DMD = disease-modifying drug; EDSS = Expanded Disability Status Scale; Gd = gadolinium; HR = hazard ratio; $\mathrm{IQR}=$ interquartile range; $\mathrm{mAB}=$ monoclonal antibody; $\mathrm{MS}=$ multiple sclerosis; $\mathrm{NfL}=$ neurofilament light chain; $\mathbf{O C B}=$ oligoclonal band.

Multiple sclerosis (MS) is a disabling disease of young adults characterized by immune-mediated focal areas of demyelination disseminated in time and space in the CNS. ${ }^{1}$ Neuropathologic evidence has highlighted that axonal damage occurs since the early phases of the disease ${ }^{2}$; thus prompt diagnosis and treatment are critical in disease management. MRI plays a major role in the diagnosis of $\mathrm{MS},{ }^{3}$ but additional clinical and paraclinical markers are known to be independent prognostic factors for the development of the disease in patients with clinically isolated syndromes (CIS) ${ }^{4,5}$ However, a disease serum marker does not exist. Neurofilament light chains (NfL) are structural elements of neurons released in the extracellular space subsequent to neuronal death, ${ }^{6,7}$ and their level has been proven to be unusually high in the CSF of patients with CIS and MS. ${ }^{8-13}$ Also, the current potential to measure NfL in patients' serum makes them the most suitable disease candidate biomarkers.

The aim of this study was to examine levels of serum NfL in patients with CIS and to assess whether there is a correlation with the potential to develop MS.

\section{Methods}

This study is part of a retrospective observational project on CIS, previously described. ${ }^{4,5,14}$ Briefly, a substantial group of patients with CIS who were admitted to our hospital between January 1 , 2000, and September 1, 2015, were involved when meeting the following inclusion criteria: (1) the occurrence for the first time of neurologic symptoms suggestive of MS that lasted more than 24 hours, and not attributable to any definitive disease subsequent to any extensive diagnostic workup, irrespective of whether or not the presence of typical white matter MRI lesions existed $^{15}$; (2) age between 15 and 55 years when the first neurologic episode occurred; (3) follow-up of more than 2 years; (4) availability of a sample of CSF and serum acquired during the hospitalization and stored at $-80^{\circ} \mathrm{C}$ since then.

\section{Standard protocol approvals, registrations, and patient consents}

Approval of the protocol was obtained from our hospital's ethical committee. Informed and signed consent was obtained from all patients at the point of admission to hospital for the carrying out of all medical examinations.

\section{Baseline examinations}

For every patient, the following information was obtained from our inpatient database and medical charts: age at disease onset, sex, form of onset (monofocal or multifocal), location of onset (spinal cord, brainstem, optic nerve, or other), onset steroid therapy, time from the onset of symptoms and the day of hospitalization, and clinical recovery (partial or complete). All the examinations were performed during the hospitalization and with a maximum interval time from each other of 7 days.

The presence of serum and CSF immunoglobulin G oligoclonal bands (OCBs) was examined by agarose isoelectric focusing together with avidin-biotin amplified double antibody peroxidase staining and immunoblotting.

Visual evoked potentials, brainstem auditory evoked potentials, somatosensory evoked potentials, and motor evoked potentials to the 4 limbs were acquired based on current guidelines ${ }^{16}$ and evaluated classified as normal or abnormal based on a conventional 4-point graded ordinal score. ${ }^{5}$ The global score ranged from 0 to 36 , with greater values representative of a severer evoked potential involvement.

MRI studies were carried out on 1.5T scanners (GE [Cleveland, $\mathrm{OH}]$ Signa $1.5 \mathrm{~T}$, Picker $1.5 \mathrm{~T}$ ) with standard head coils and consisted of axial T1, T2, and contrast-enhanced T1 sequences covering the entire brain, with thickness of slices ranging between 3 and $5 \mathrm{~mm}$. Fluid-attenuated inversion recovery scans were utilized to improve confidence levels in the identification of lesions. Of note, double inversion recovery sequences for detecting cortical lesions were not part of our routine clinical practice for the years considered. Spinal cord MRIs were carried out in an estimated $50 \%$ of patients. Images of the brain were logged as normal if there was an absence of subclinical lesions, with size or shape consistent with demyelination criteria (i.e., lesions with size $<3 \mathrm{~mm}$ in long axis were not considered in the analysis).

\section{Measurement of NfL levels}

Collection of serum and CSF samples was by standard methods and samples were stored in cryogenic vials at $-80^{\circ} \mathrm{C}$. NfL levels were measured using an electrochemiluminescence immunoassay, which had been previously validated. ${ }^{17}$ Antibodies used were as follows: capture monoclonal antibody $(\mathrm{mAB})$ 47:3 and biotinylated detector $\mathrm{mAB}$ 2:1 (UmanDiagnostics, Umea, Sweden). The detection reagent was MSD SULFO-TAGTM labeled streptavidin. Bovine lyophilized NfL from UmanDiagnostics was utilized to generate standards ranging from 0 to $10,000 \mathrm{pg} / \mathrm{mL}$. The mean intra-assay and interassay coefficients of variation were, respectively, $5.4 \%$ and $9.9 \%$. A level of 
serum NfL of $3.91 \mathrm{pg} / \mathrm{mL}$ was the analytical sensitivity of our assay, defined as the concentration of the lowest calibrator with accuracy $80 \%-120 \%$ and coefficients of variation of duplicate determination $\leq 20 \%$. $^{18}$

\section{Outcomes and follow-up}

Follow-up data were obtained from medical records and the time between the occurrence of CIS and the last neurologic visit was used as the follow-up duration period.

A clinically definite MS (CDMS) diagnosis was carried out after new signs or symptoms appeared a minimum of 1 month subsequent to the onset of CIS and only after the exclusion of other diagnoses. ${ }^{15}$ According to the 2017 diagnostic criteria (McDonald $2017 \mathrm{MS}$ ), a diagnosis of MS was made when the presence of CSF oligoclonal bands and, at any time, the simultaneous presence of gadolinium (Gd)-enhancing and nonenhancing lesions, or new T2 or Gd-enhancing lesions on follow-up MRI, were seen in patients who had lesions in at least 2 of the 4 typical areas of the CNS. ${ }^{1}$

All patients developing CDMS were given diseasemodifying drugs (DMDs) following their second clinical attack, while 100 patients began immunomodulatory

Table 1 Baseline characteristics of patients who developed McDonald 2017 multiple sclerosis (MS) and patients who did not $^{a}$

\begin{tabular}{|c|c|c|c|}
\hline Characteristic & All patients $(n=222)$ & MS ( $n=152)$ & No MS $(n=70)$ \\
\hline Follow-up, mo, mean \pm SD & $100.6 \pm 58.0$ & $103.4 \pm 59.9$ & $94.4 \pm 53.9$ \\
\hline Age at blood collection, $y$, mean \pm SD & $33.0 \pm 9.4$ & $31.5 \pm 9.2$ & $36.2 \pm 9.2$ \\
\hline \multicolumn{4}{|l|}{ Sex, n (\%) } \\
\hline Female & $149(67)$ & $101(66)$ & $48(69)$ \\
\hline Male & $73(33)$ & $51(34)$ & $22(31)$ \\
\hline \multicolumn{4}{|l|}{ Type of onset, $\mathbf{n}(\%)$} \\
\hline Monofocal & $192(86)$ & $126(83)$ & $66(94)$ \\
\hline Brainstem & $41(21)$ & $21(17)$ & $20(30)$ \\
\hline Optic nerve & $65(34)$ & $46(37)$ & $19(29)$ \\
\hline Spinal cord & $81(42)$ & $56(44)$ & $25(38)$ \\
\hline Other & $5(3)$ & $3(2)$ & $2(3)$ \\
\hline Multifocal & $30(14)$ & $26(17)$ & $4(6)$ \\
\hline Expanded Disability Status Scale score, median (IQR) & $1.5(1.0-2.0)$ & $1.5(1.0-2.0)$ & $1.5(1.0-2.0)$ \\
\hline \multicolumn{4}{|l|}{ Clinical episode outcome, $\mathbf{n}(\%)$} \\
\hline Complete recovery & $105(47)$ & $72(47)$ & $33(47)$ \\
\hline Partial recovery & $117(53)$ & $80(53)$ & $37(53)$ \\
\hline Duration of symptoms, d, median (IQR) & $26.4(20.1-30.0)$ & $26.0(19.3-30.0)$ & $28.8(20.8-30.0)$ \\
\hline \multicolumn{4}{|l|}{ T2 lesions at brain MRI, n (\%) } \\
\hline $0-1$ & $30(14)$ & $7(5)$ & $23(33)$ \\
\hline $2-9$ & $90(41)$ & $54(36)$ & $36(51)$ \\
\hline$>9$ & $102(46)$ & $91(60)$ & $11(16)$ \\
\hline Presence of gadolinium-enhancing lesions, n (\%) & $97(44)$ & $82(54)$ & $15(21)$ \\
\hline Presence of CSF oligoclonal bands, $\mathbf{n}(\%)$ & $161(73)$ & $128(84)$ & $33(47)$ \\
\hline Multimodal evoked potentials score, median (IQR) & $1(0.0-3.0)$ & $2(0-3)$ & $1(0-3)$ \\
\hline CSF neurofilament light chain levels, pg/mL, median (IQR) & $731.3(346.9-1,194.6)$ & $972.8(442.2-1,291.8)$ & $510.4(253.5-882.9)$ \\
\hline Serum neurofilament light chain levels, pg/mL, median (IQR) & $22.0(11.6-40.4)$ & $30.2(16.4-48.7)$ & $9.7(5.5-18.1)$ \\
\hline
\end{tabular}


therapy after the demonstration of new subclinical lesions, according to the available MS diagnostic criteria. Among patients who developed MS, disability worsening was defined as a documented increase in neurologic disability $(\geq 1$ point increase in the Expanded Disability Status Scale (EDSS) score, or $\geq 0.5$ point increase for patients with a baseline score of $\geq 5.5$, confirmed in a second visit 3 months later). ${ }^{19}$

\section{Statistical analysis}

Differences between groups of normally and non-normally distributed variables were analyzed with $t$ tests and MannWhitney $U$ tests, respectively. Analysis of categorical variables was performed using $\chi^{2}$ tests.

Multiple regression analysis was carried out to establish what factors influenced NfL baseline levels.

The calculation of follow-up person-years was from baseline until the diagnosis of CDMS, reaching the follow-up period's end, or death. Cox proportional hazards models were utilized to approximate hazard ratios (HRs) and $95 \%$ confidence intervals (CIs) for development of CDMS and MRI MS. To make allowances for NfL level nonlinear association with the time to event, restricted cubic-spline Cox proportional hazard models were used. ${ }^{20}$ The results are presented as smoothed plots of HRs and 95\% CIs for CDMS and McDonald 2017 MS.

NfL level extremes were analyzed by subject categorization into the lowest and highest $10 \%$ and $25 \%$ of the NfL concentration distribution. HRs were computed using basic and multivariable models. The base models included NfL levels, age at onset, and sex of patients (and DMD for time to CDMS analysis). Adjusted models also included all significant prognostic factors for CDMS and McDonald 2017 MS, plus covariates influencing NfL levels - such as time from onset to blood sampling. The proportional hazard assumptions were confirmed using Shoenfeld residuals. Assessment of interactions was performed to test for a product term coefficient created from the covariates involved. Nomograms were utilized for the assessment of the prognostic serum NfL

Table 2 Baseline characteristics of patients by serum neurofilament light chain (NfL) levels, $\mathrm{pg} / \mathrm{mL}$

\begin{tabular}{|c|c|c|c|c|c|}
\hline & \multicolumn{5}{|c|}{ Serum NfL levels } \\
\hline & $\begin{array}{l}<10 \text { th } \\
\text { percentile }\end{array}$ & $\begin{array}{l}<25 \text { th } \\
\text { percentile }\end{array}$ & $\begin{array}{l}\text { 25th-75th } \\
\text { percentile }\end{array}$ & $\begin{array}{l}>75 \text { th } \\
\text { percentile }\end{array}$ & $\begin{array}{l}>90 \text { th } \\
\text { percentile }\end{array}$ \\
\hline Serum NfL levels, pg/mL & $<5.6$ & $<11.6$ & $11.6-40.2$ & $>40.4$ & $>107.4$ \\
\hline Age at blood collection, $y$, mean $\pm S D$ & $36.3 \pm 7.2$ & $33.7 \pm 8.4$ & $32.5 \pm 9.2$ & $31.8 \pm 9.7$ & $28.8 \pm 10.7$ \\
\hline Time from onset to hospital, mo, mean \pm SD & $2.8 \pm 6.2$ & $2.5 \pm 2.8$ & $2.3 \pm 2.2$ & $1.9 \pm 1.8$ & $1.6 \pm 2.0$ \\
\hline \multicolumn{6}{|l|}{ Sex } \\
\hline Female, n (\%) & $11(48)$ & $30(58)$ & $77(69)$ & $42(69)$ & $18(78)$ \\
\hline Male, n (\%) & $12(52)$ & $26(42)$ & $34(31)$ & $13(31)$ & $5(22)$ \\
\hline \multicolumn{6}{|l|}{ Type of onset, n (\%) } \\
\hline Monofocal & $22(96)$ & $51(93)$ & $90(83)$ & $51(100)$ & $18(78)$ \\
\hline Brainstem & $5(23)$ & $10(20)$ & $16(18)$ & $15(29)$ & $6(33)$ \\
\hline Optic nerve & $6(27)$ & $16(31)$ & $27(30)$ & $22(43)$ & $6(33)$ \\
\hline Spinal cord & $11(50)$ & $25(49)$ & $45(50)$ & $11(22)$ & $4(22)$ \\
\hline Other & $0(0)$ & $0(0)$ & $2(2)$ & $3(6)$ & $0(0)$ \\
\hline Multifocal & $1(4)$ & $5(9)$ & $21(19)$ & $4(2)$ & $3(22)$ \\
\hline \multicolumn{6}{|l|}{ T2 lesions at brain MRI, $n(\%)$} \\
\hline $0-1$ & $9(39)$ & $16(29)$ & $12(11)$ & $2(4)$ & $1(4)$ \\
\hline $2-9$ & $10(43)$ & $25(46)$ & $57(52)$ & $8(16)$ & $3(13)$ \\
\hline$>9$ & $4(17)$ & $15(25)$ & $42(37)$ & $45(80)$ & $19(83)$ \\
\hline Presence of gadolinium-enhancing lesions, $n$ (\%) & $3(13)$ & $11(20)$ & $43(39)$ & $43(78)$ & $19(83)$ \\
\hline Presence of CSF oligoclonal bands, n (\%) & $11(48)$ & $29(52)$ & $90(82)$ & $42(76)$ & $16(70)$ \\
\hline $\begin{array}{l}\text { Multimodal evoked potentials score, median } \\
\text { (interquartile range) }\end{array}$ & $2.0(0.0-3.0)$ & $1.0(0.0-3.0)$ & $1.0(0.0-3.0)$ & $0.0(0.0-3.0)$ & $0.0(0.0-3.5)$ \\
\hline
\end{tabular}


contribution to known MS prognostic markers. Using 200 resamples, bootstrap validation was utilized to gauge the models' performance and a concordance index was subsequently calculated. ${ }^{21,22}$ In each event where data were absent, analyses were restricted to only those patients for whom there was complete data. $p$ Values that were less than 0.05 were deemed statistically significant. Computing environment $\mathrm{R}$ was used for all statistical analyses ( $\mathrm{R}$ Development Core Team, 2005).

\section{Data availability}

Anonymized data will be shared by request from any qualified investigator.

\section{Results}

We identified 222 patients with a CIS diagnosis who met the inclusion criteria (mean follow-up 100.6 \pm 58.0 months). At 2 years, 45 patients (20\%) developed CDMS and 141 patients (63.5\%) developed McDonald 2017 MS, while at 5 years (136 patients had a minimum of 5 consecutive years of follow-up), 70 patients developed CDMS (51.5\%) and 108 (79.4\%) had McDonald 2017 MS.

Table 1 summarizes the principal clinical and demographic patient characteristics at baseline. Patients who subsequently developed CDMS were younger, had a greater likelihood of reporting multifocal symptoms, and had a greater chance of having CSF OCBs, Gd-enhancing lesions, plus a greater number of $\mathrm{T} 2$ lesions at brain MRI than those patients who had not converted to MS.

\section{Predictor of baseline serum NfL levels}

The median baseline level of serum NfL was $22.0 \mathrm{pg} / \mathrm{mL}$ (interquartile range [IQR] 11.6-40.4), with CIS patients subsequently developing MS having higher levels (median 30.2, IQR $16.4-48.7 \mathrm{pg} / \mathrm{mL}$ ) than patients who did not develop MS (median 9.7, IQR 5.5-18.1 pg/mL, $p<0.001$ ) (table 2). Median CSF NfL level in CIS patients was 731.3 (IQR 346.9-1,194.6) $\mathrm{pg} / \mathrm{mL}$ and they were directly related with serum levels $(r=0.62, p<0.001)$, particularly in patients with Gd-enhancing lesions $(r=0.71, p<0.001$; figure e-1 [doi.org/10.5061/dryad.t95q4kg]).

For the univariate analysis, patients' age at blood sample, time interval from the clinical onset, baseline EDSS, Gd-enhancing lesions, and a high T2 lesion load were all associated with baseline serum NfL levels. The results were confirmed with multivariate analysis, with NfL levels being lower with an increase in time from the onset of symptoms ( $\beta-2.0,95 \% \mathrm{CI}$ -3.9 to $-0.1, p=0.04$ ), and being higher in patients with the presence of a high T2 lesion load and Gd-enhancing lesions at baseline MRI ( $\beta$ 35.4, 95\% CI 17.1-53.8, $p<0.001$; and $\beta$ 22.9, 95\% CI 10.8-35.1, $p<0.001$; table 3, figure 1).

\section{Baseline serum NfL levels and risk of MS}

In the survival analysis, an association was seen between baseline serum NfL levels and risk of both CDMS and McDonald 2017 MS (figure 2). Patients with very high (>90th percentile) and high ( $>75$ th percentile) NfL levels were especially at risk of CDMS, whereas a lowering of the HRs of both CDMS and McDonald 2017 MS was seen in patients with low levels of serum NfL. Analysis of risks in the low and high ends of the range of serum NfL levels confirmed these graphical findings (table 4),

Table 3 Predictors of serum neurofilament light chain levels, ${ }^{a} \mathrm{pg} / \mathrm{mL}$

\begin{tabular}{|c|c|c|c|c|c|c|}
\hline & \multicolumn{3}{|c|}{ Univariate analysis } & \multicolumn{3}{|c|}{ Multivariate analysis } \\
\hline & Coefficient & $95 \% \mathrm{Cls}$ & $p$ & Coefficient & $95 \%$ Cls & $p$ \\
\hline Age at blood collection & -0.9 & -1.5 to -0.2 & 0.01 & -0.5 & -1.1 to 0.1 & 0.13 \\
\hline Time from onset to hospital & -2.1 & -4.2 to -0.1 & 0.04 & -2.0 & -3.9 to -0.1 & 0.04 \\
\hline Sex, male vs female & -8.2 & -21.8 to 5.3 & 0.23 & - & - & - \\
\hline Type of onset, multifocal vs monofocal & 15.4 & -4.4 to 35.2 & 0.12 & - & - & - \\
\hline EDSS at onset & 7.3 & -0.1 to 14.6 & 0.05 & - & - & - \\
\hline \multicolumn{7}{|l|}{ T2 lesions at brain MRI } \\
\hline $2-9$ vs $0-1$ & 11.5 & -7.5 to 30.5 & 0.24 & 7.9 & -10.2 to 26.2 & 0.39 \\
\hline$>9$ vs $0-1$ & 39.5 & 20.7 to 58.4 & $<0.001$ & 35.4 & 17.1 to 53.8 & $<0.001$ \\
\hline Presence of gadolinium-enhancing lesions & 29.8 & 17.4 to 42.3 & $<0.001$ & 22.9 & 10.8 to 35.1 & $<0.001$ \\
\hline Presence of CSF oligoclonal bands & 4.4 & -9.9 to 18.6 & 0.54 & - & - & - \\
\hline Multimodal evoked potentials score & 0.2 & -2.1 to 1.8 & 0.87 & - & - & - \\
\hline
\end{tabular}

Abbreviations: $\mathrm{Cl}$ = confidence interval; EDSS = Expanded Disability Status Scale.

a Univariate and multivariate regression analysis with AIC-based backward selection was performed to determine the factors significantly influencing baseline NfL levels. 

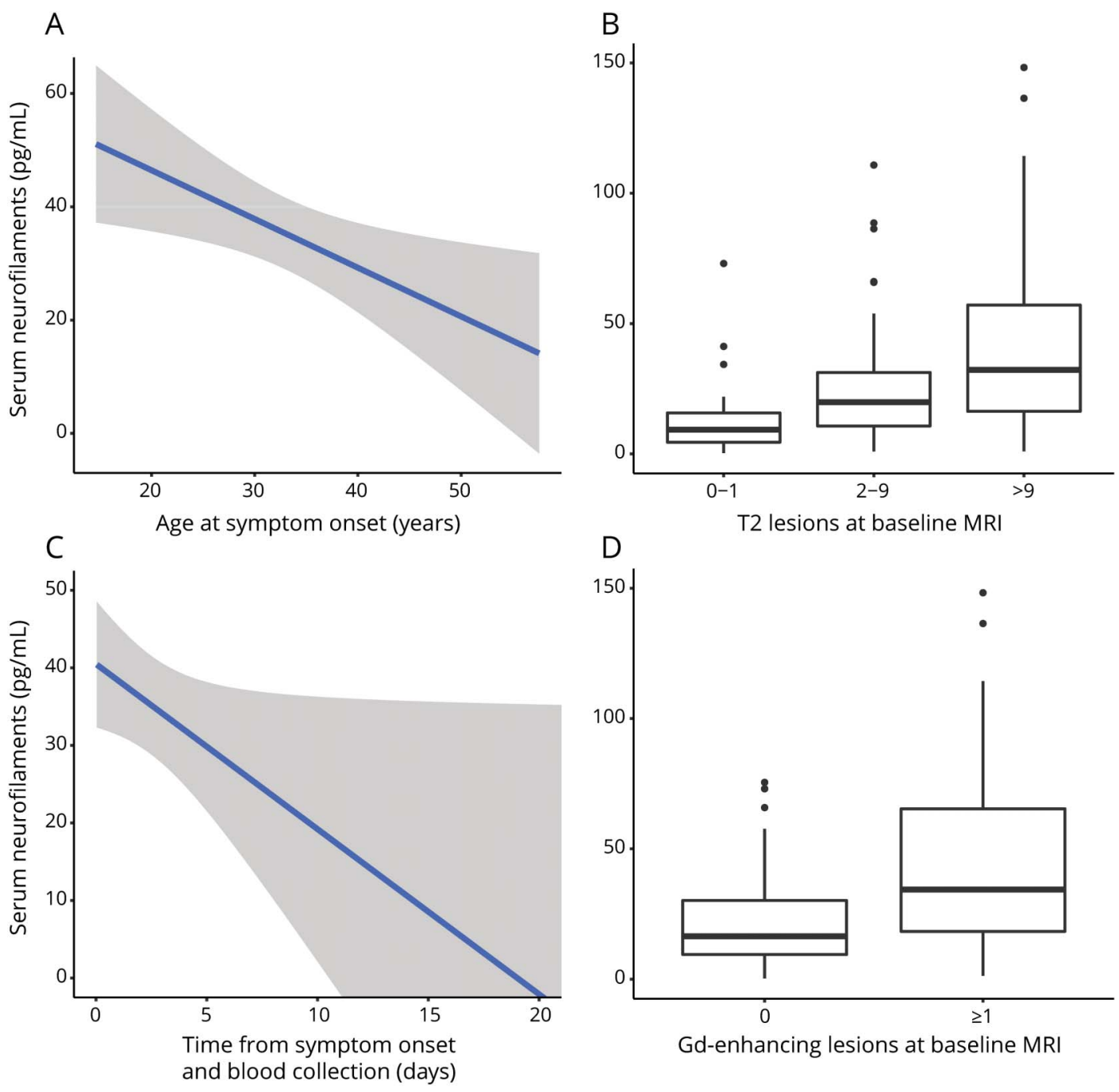

with a threefold and a twofold decrease of both CDMS and McDonald $2017 \mathrm{MS}$ risk in patients with low and very low NfL levels (HR [95\% CI] for CDMS: 0.25 [0.11-0.58] and 0.09 [0.01-0.64], respectively; $\mathrm{HR}$ [95\% CI] for McDonald 2017 MS: 0.21 [0.12-0.37] and 0.34 [0.16-0.76], respectively).

This association remained unchanged after adjustments were made for the following covariates: presence of CSF OCBs, Gd-enhancing lesions, and a high T2 lesion load at baseline MRI (HRs of CDMS for very low $[<10$ th percentile $]$ and low [ $<25$ th percentile $]$ NfL levels were 0.14 [0.02-1.05] and 0.40 [0.17-0.94], respectively; HRs [95\% CI] for McDonald 2017 MS were 0.18 [0.06-0.59] and 0.33 [0.19-0.60], respectively; table 4).

Of note, 35 patients (23\%) among the 152 who developed MS experienced EDSS worsening during follow-up, but no association was found between baseline NfL levels and disability progression.

\section{Development of prognostic nomograms}

Nomograms were developed to help identify those patients who have a high risk for developing CDMS and McDonald 2017 MS based on the models' beta coefficients (figure 3). These nomograms reveal serum NfL's prognostic contribution to already known prognostic markers in CIS patients. At 2 and 5 years, serum NfL seems to be especially helpful in stratifying the risk of disease in patients who have a modest lesion load in their baseline brain MRI.

In addition, a bootstrap-corrected concordance index (cindex) was used to assess the proposed models' performance. The c-indexes calculated were 0.65 and 0.85 for the final models, predicting CDMS and McDonald 2017 MS, respectively, thus the prognostic models could be considered acceptably accurate. In the calibration plots (figure e-2, doi. org/10.5061/dryad.t95q4kg), points in the proximity of the 45-degree line reveal solid agreement between observed and predicted outcomes. 

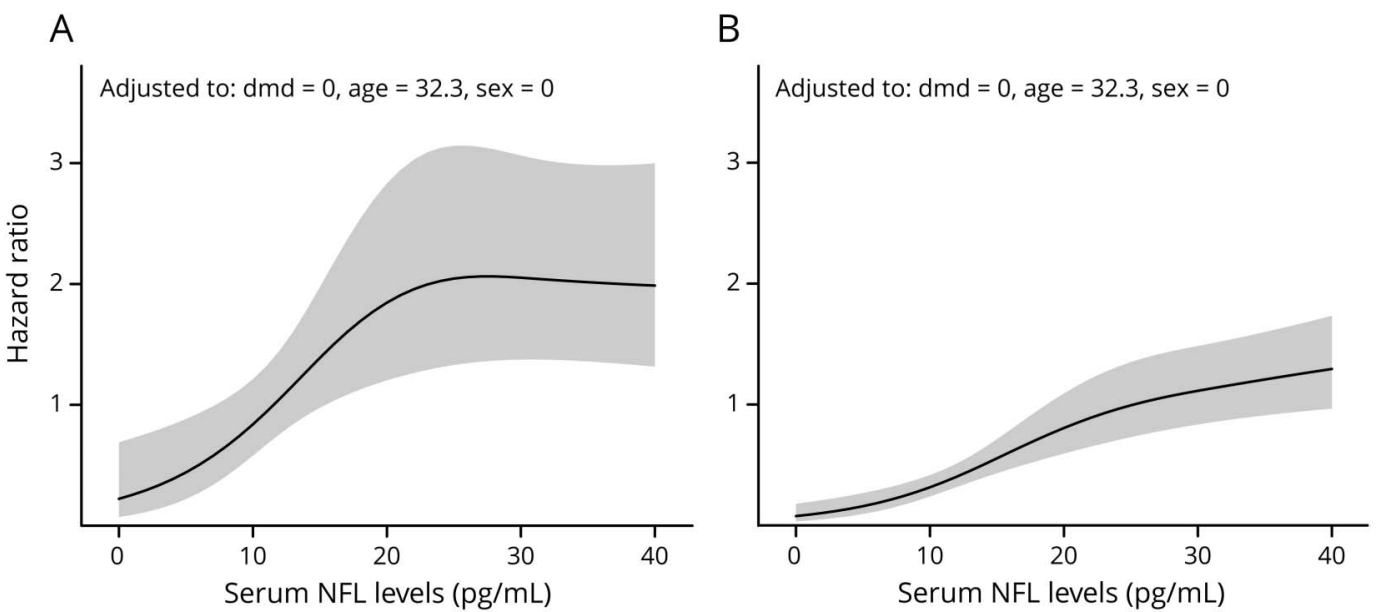

Smoothed plot of hazard ratios for clinically definite multiple sclerosis (A) and McDonald 2017 multiple sclerosis (B) according to serum neurofilament light (NfL) levels.

\section{Discussion}

Several studies have shown that axonal damage occurs from the very early phases of MS. ${ }^{2}$ Therefore, early diagnosis has been the major objective of evolving diagnostic criteria for the disease. ${ }^{1}$ MRI has played a key role in this issue since its introduction. Several other biomarkers are also associated with the risk of developing MS independently of MRI, among which the presence and number of CSF OCBs have been revealed to be crucial for the stratification of risk at patient level. ${ }^{4} \mathrm{NfL}$ level in the CSF has been previously shown to be a risk factor for conversion to MS in CIS patients, ${ }^{8-13}$ but the recent possibility of measuring this structural protein also in the serum would make NfL an ideal and easy-to-measure biomarker for a more accurate prognosis at the time of the first clinical demyelinating event.
Several studies ${ }^{8-11}$ have already reported higher levels of CSF NfL in CIS patients when compared to healthy controls, and in a recent study ${ }^{13}$ they have been shown to be prognostic both for CDMS and McDonald 2010 MS. A linear relationship between CSF and serum NfL has also been previously reported, ${ }^{23}$ and we have highlighted here that serum NfL levels mirror in particular CSF levels in patients with blood-brain barrier damage as evidenced by the presence of Gd-enhancing lesions. The main result of the current study is the prognostic value of serum NfL both for CDMS and McDonald 2017 MS, here reported for the first time. As expected, we observed significant associations between serum $\mathrm{NfL}$ and patient age, disability, and lesion load at baseline, but we have also found NfL levels are influenced by inflammatory measures, with serum NfL levels higher in patients evaluated close to the onset of a clinical relapse or with Gd-enhancing

Table 4 Hazard ratios (95\% confidence intervals) of clinically definite multiple sclerosis (CDMS) and multiple sclerosis (MS) according to the 2017 McDonald criteria (2017 MS) by categories of serum neurofilament light chains levels ${ }^{a}$

\begin{tabular}{|c|c|c|c|c|c|}
\hline & \multicolumn{5}{|c|}{ Serum neurofilament light chain levels } \\
\hline & $<10$ th percentile & $<25$ th percentile & 25th-75th percentile & >75th percentile & >90th percentile \\
\hline \multicolumn{6}{|l|}{ Overall CDMS risk } \\
\hline Base model & $0.09(0.01-0.64)^{\mathrm{b}}$ & $0.25(0.11-0.58)^{c}$ & Reference & $1.13(0.68-1.89)$ & $1.22(0.60-2.46)$ \\
\hline Adjusted model & $0.14(0.02-1.05)^{d}$ & $0.40(0.17-0.94)^{d}$ & Reference & $1.11(0.63-1.95)$ & $1.24(0.57-2.73)$ \\
\hline \multicolumn{6}{|c|}{ Overall 2017 MS risk } \\
\hline Base model & $0.34(0.16-0.76)^{c}$ & $0.21(0.12-0.37)^{c}$ & Reference & $2.03(1.43-2.89)^{c}$ & $2.19(1.32-3.62)^{c}$ \\
\hline Adjusted model & $0.18(0.06-0.59)^{\mathrm{b}}$ & $0.33(0.19-0.60)^{\mathrm{b}}$ & Reference & $1.64(1.10-2.44)^{b}$ & $1.62(0.98-2.81)^{d}$ \\
\hline \multicolumn{6}{|c|}{$\begin{array}{l}\text { a Hazard ratios and 95\% confidence intervals were estimated by Cox proportional hazards regression. Base model for CDMS was adjusted for age, sex, and } \\
\text { time of follow-up on disease-modifying therapy, while base model for } 2017 \text { MS was adjusted for age and sex. Multivariable adjusted models were adjusted } \\
\text { also for CSF oligoclonal bands, gadolinium, and T2 lesions at baseline MRI. } \\
\text { b } p<0.01 \text {. } \\
\text { c } p<0.001 . \\
\text { d } p<0.05 \text {. }\end{array}$} \\
\hline
\end{tabular}


Figure 3 Nomograms for predicting 2-year probabilities

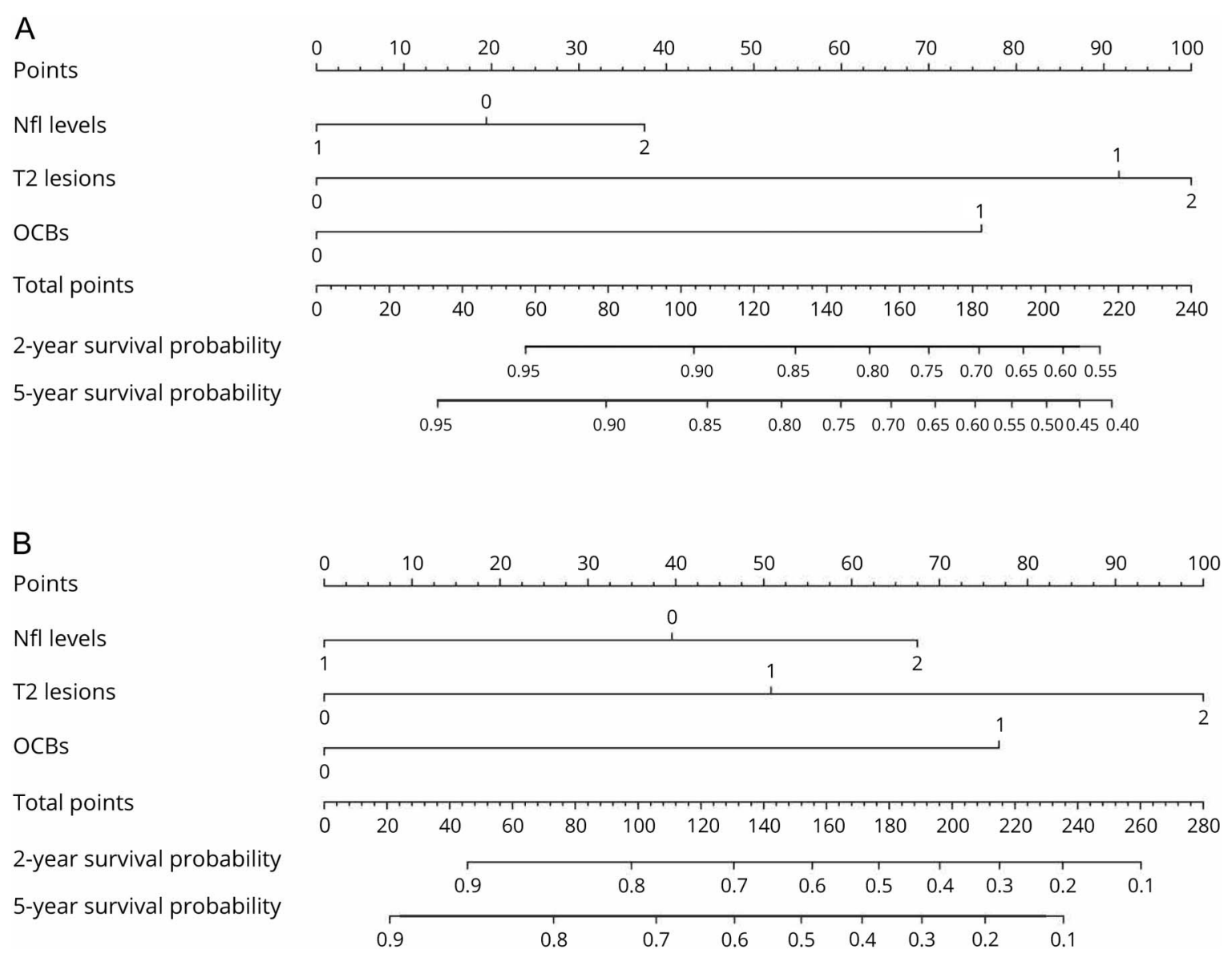

Nomograms for predicting 2-year probabilities for clinically definite multiple sclerosis (MS) (A) and for McDonald 2017 MS (B) according to baseline neurofilament light (NfL), CSF, and brain MRI status.Categories for NfL: $0(11.6-40.2 \mathrm{pg} / \mathrm{mL}), 1(<11.6 \mathrm{pg} / \mathrm{mL}), 2(>40.2 \mathrm{pg} / \mathrm{mL})$. Categories for CSF oligoclonal bands (OCBs): 0 (absent), 1 (present). Categories for brain MRI T2 lesions: 0 (0 lesions), 1 (1-3 lesions), 2 (4-9 lesions), 3 ( $>9$ lesions). To obtain the 2-year and 5 year survival probabilities, calculate trough use of the first row the points for every patient characteristic listed, then drop a vertical line from the total points row to the probabilities rows.

lesions at brain MRI. A high T2 lesion load and the presence of enhancing lesions at baseline MRI are signs of persistent or recurrent active disease and therefore predictors of a shorter time to a second relapse, and the fact that $\mathrm{NfL}$ are also higher in these patients may confirm the aggressive nature of the underlying pathologic process and explain why the serum biomarkers also resulted prognostic for a second attack.

Our results are in line with previous studies that show an association between serum NfL and clinical and MRI inflammatory measures. ${ }^{24}$ This also could be the main reason why we have found no associations between NfL levels and future disability evaluated by EDSS. Except for two, ${ }^{13,23}$ most studies ${ }^{24-26}$ have shown a correlation between NfL and future disability, and NfL are now regarded as a promising serologic biomarker for monitoring and predicting neurodegeneration. However, in all these studies, the analyses included different MS phenotypes, observing the strongest correlations with current and future EDSS in progressive forms. Furthermore, in all the studies, association between signs of active disease and NfL was observed, and repeated inflammatory attacks may yield to increased disability in later stages of the disease. As such, serum NfL may have a different role as a biomarker according to the phase of the disease the patient is experiencing: measured peak levels may be a marker of recent destructive effects related to acute inflammatory activity, while steady-state levels may reflect both basal CNS metabolic processes as well as chronic inflammatory and neurodegenerative processes.

In our study, serum NfL was predictive of CDMS and McDonald 2017 MS independently of known major prognostic factors such as T2 lesion load and CSF OCBs, allowing for a more accurate discrimination of the model and the provision of clinical relevance to our results.

The simultaneous evaluation of CSF and serum NfL as well as the long follow-up and the real-world group of patients are the major strengths of our study and our results ought to be a better representation of clinical practice. The use of restricted cubic spline analysis and the nomogram derived further strengthens our study as they allow a more detailed 
analysis of the data and provide clinicians with both a useful and practical tool for individual prognostic prediction. Our results have been internally validated using bootstrap, ${ }^{27}$ but external validation is still essential prior to our nomograms being generalized to a new population of patients. ${ }^{28} \mathrm{~A}$ lack in knowledge of important covariates including MRI lesion volumes as well as cortical lesions plus the retrospective nature of the work could be seen as limitations hindering the drawing of definitive conclusions. Multicentric prospective studies that will involve a substantial number of CIS patients and will also take into account all potential NfL confounding factors are required for confirmation of these preliminary findings.

\section{Author contributions}

G.D. Costa conceived and designed the study and performed the data collection, statistical analyses, literature research, discussion of the findings, and writing of the manuscript. V. Martinelli conceived and designed the study, supervised the process of data collection and statistical analyses, and performed discussion of the findings. R. Furlan managed the biobank used in this study, supervised the process of data collection and statistical analyses, and performed discussion of the findings. G. Comi conceived and designed the study, supervised the process of data collection and statistical analyses, and performed discussion of the findings. All authors revised, edited, and approved the manuscript for submission.

\section{Study funding}

This research was partially funded by a grant from the Italian Ministry of Health (RF 2011-02349698).

\section{Disclosure}

G. Dalla Costa reports no disclosures relevant to the manuscript. V. Martinelli received honoraria for consulting services or speaking activity from Biogen, Merck, Novartis, TEVA, Almirall, and Sanofi-Genzyme. F. Sangalli reports no disclosures relevant to the manuscript. L. Moiola received honoraria for speaking in scientific meetings and for advisory board from Biogen TEVA, Sanofi-Genzyme, and Merck. B. Colombo and M. Radaelli report no disclosures relevant to the manuscript. R. Furlan received honoraria for speaker activities from Biogen, Merck, Novartis, Roche, and Teva. L. Leocani received honoraria for consulting services and/or speaking activity from Biogen, Novartis, and Excemed. G. Comi received personal compensation for consulting services and/or speaking activities from Novartis, Teva, Sanofi, Sanofi-Genzyme, Merck, Biogen, Excemed, Serono Symposia International Foundation, Roche, Almirall, Receptos, Celgene, and Forward Pharma. Go to Neurology.org/N for full disclosures.

\section{Publication history}

Received by Neurology February 14, 2018. Accepted in final form December 14, 2018.

\section{References}

1. Thompson AJ, Banwell BL, Barkhof F, et al. Diagnosis of multiple sclerosis: 2017 revisions of the McDonald criteria. Lancet Neurol 2018;17:162-173.

2. Trapp BD, Peterson J, Ransohoff RM, et al. Axonal transection in the lesions of multiple sclerosis. N Engl J Med 1998;338:278-285.

3. Young IR, Hall AS, Pallis CA, et al. Nuclear magnetic resonance imaging of the brain in multiple sclerosis. Lancet 1981;2:1063-1066.

4. Dalla Costa G, Passerini G, Messina MJ, et al. Clinical significance of the number of oligoclonal bands in patients with clinically isolated syndromes. J Neuroimmunol 2015;289:62-67.

5. Martinelli V, Dalla Costa G, Messina MJ, et al. Multiple biomarkers improve the prediction of multiple sclerosis in clinically isolated syndromes. Acta Neurol Scand 2017; 136:454-461.

6. Fuchs E, Cleveland DW. A structural scaffolding of intermediate filaments in health and disease. Science 1998;279:514-519.

7. Yabe JT, Chylinski T, Wang FS, et al. Neurofilaments consist of distinct populations that can be distinguished by C-terminal phosphorylation, bundling, and axonal transport rate in growing axonal neurites. J Neurosci 2001;21:2195-2205.

8. Teunissen CE, Iacobaeus $\mathrm{E}$, Khademi M, et al. Combination of CSF N-acetylaspartate and neurofilaments in multiple sclerosis. Neurology 2009;72:1322-1329.

9. Khalil M, Enzinger C, Langkammer C, et al. CSF neurofilament and $\mathrm{N}$-acetylaspartate related brain changes in clinically isolated syndrome. Mult Scler 2013;19:436-442.

10. Modvig S, Degn M, Horwitz H, et al. Relationship between cerebrospinal fluid biomarkers for inflammation, demyelination and neurodegeneration in acute optic neuritis. PLoS One 2013;8:e77163.

11. Hakansson I, Tisell A, Cassel P, et al. Neurofilament light chain in cerebrospinal fluid and prediction of disease activity in clinically isolated syndrome and relapsingremitting multiple sclerosis. Eur J Neurol 2017;24:703-712.

12. Disanto G, Adiutori R, Dobson R, et al. Serum neurofilament light chain levels are increased in patients with a clinically isolated syndrome. J Neurol Neurosurg Psychiatry 2016;87:126-129.

13. Arrambide G, Espejo C, Eixarch H, et al. Neurofilament light chain level is a weak risk factor for the development of MS. Neurology 2016;87:1076-1084.

14. Martinelli V, Dalla Costa G, Colombo B, et al. Vitamin D levels and risk of multiple sclerosis in patients with clinically isolated syndromes. Mult Scler 2014;20:147-155.

15. Poser CM, Paty DW, Scheinberg L, et al. New diagnostic criteria for multiple sclerosis: guidelines for research protocols. Ann Neurol 1983;13:227-231.

16. Deuschl G, Eisen A. Recommendations for the practice of clinical neurophysiology: guidelines of the International Federation of Clinical Neurophysiology. Electroencephalogr Clin Neurophysiol 1999;52:192-211.

17. Gaiottino J, Norgren N, Dobson R, et al. Increased neurofilament light chain blood levels in neurodegenerative neurological diseases. PLoS One 2013;8:e75091.

18. Kuhle J, Barro C, Andreasson, et al. Comparison of three analytical platforms for quantification of the neurofilament light chain in blood samples: ELISA, electrochemiluminescence immunoassay and Simoa. Clin Chem Lab Med 2016;54:1655-1661.

19. Rudick R, Antel J, Confavreux C, et al. Recommendations from the National Multiple Sclerosis Society Clinical Outcomes Assessment Task Force. Ann Neurol 1997;42:379-382.

20. Harrell FE Jr. General Aspects of Fitting Regression Models: Regression Modeling Strategies. New York: Springer Science+Business Media; 2001:23.

21. Iasonos A, Schrag D, Raj GV, Panageas KS. How to build and interpret a nomogram for cancer prognosis. J Clin Oncol 2008;26:1364-1370.

22. Shen W, Sakamoto N, Yang L. Prognostic models to predict overall and cause-specific survival for patients with middle ear cancer: a population-based analysis. BMC Cancer 2014;14:554.

23. Kuhle J, Barro C, Disanto G, et al. Serum neurofilament light chain in early relapsing remitting MS is increased and correlates with CSF levels and with MRI measures of disease severity. Mult Scler 2016;22:1550-1559.

24. Disanto G, Barro C, Benkert P, et al. Serum neurofilament light: a biomarker of neuronal damage in multiple sclerosis. Ann Neurol 2017;81:857-870.

25. Malmestrom C, Haghighi S, Rosengren L, Andersen O, Lycke J. Neurofilament light protein and glial fibrillary acidic protein as biological markers in MS. Neurology 2003; 61:1720-1725.

26. Norgren N, Sundstrom P, Svenningsson A, Rosengren L, Stigbrand T, Gunnarsson M. Neurofilament and glial fibrillary acidic protein in multiple sclerosis. Neurology 2004; 63:1586-1590.

27. Moons KG, Kengne AP, Woodward M, et al. Risk prediction models: I: development, internal validation, and assessing the incremental value of a new (bio)marker. Heart 2012;98:683-690.

28. Moons KG, Kengne AP, Grobbee DE, et al. Risk prediction models: II: external validation, model updating, and impact assessment. Heart 2012;98:691-698. 


\section{Neurology}

\section{Prognostic value of serum neurofilaments in patients with clinically isolated syndromes}

Gloria Dalla Costa, Vittorio Martinelli, Francesca Sangalli, et al.

Neurology 2019;92;e733-e741 Published Online before print January 11, 2019

DOI 10.1212/WNL.0000000000006902

\section{This information is current as of January 11, 2019}

\section{Updated Information \&} Services

References

Citations

Permissions \& Licensing

Reprints including high resolution figures, can be found at: http://n.neurology.org/content/92/7/e733.full

This article cites 27 articles, 10 of which you can access for free at: http://n.neurology.org/content/92/7/e733.full\#ref-list-1

This article has been cited by 1 HighWire-hosted articles: http://n.neurology.org/content/92/7/e733.full\#\#otherarticles

Information about reproducing this article in parts (figures,tables) or in its entirety can be found online at:

http://www.neurology.org/about/about_the_journal\#permissions

Information about ordering reprints can be found online:

http://n.neurology.org/subscribers/advertise

Neurology ${ }^{\circledR}$ is the official journal of the American Academy of Neurology. Published continuously since 1951, it is now a weekly with 48 issues per year. Copyright Copyright (C) 2019 The Author(s). Published by Wolters Kluwer Health, Inc. on behalf of the American Academy of Neurology.. All rights reserved. Print ISSN: 0028-3878. Online ISSN: 1526-632X.

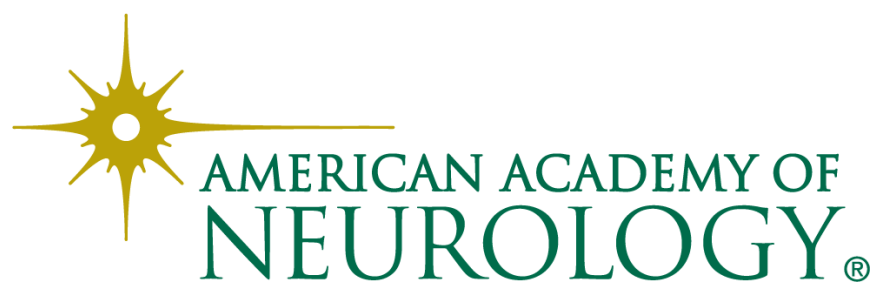

